\title{
The Hoffmeister asteroid family
}

\author{
V. Carruba, ${ }^{1 \star}$ B. Novaković ${ }^{2}$ and S. Aljbaae ${ }^{1}$ \\ ${ }^{1}$ UNESP, Universidade Estadual Paulista, Grupo de Dinâmica Orbital e Planetologia, Guaratinguetá, SP 12516-410, Brazil \\ ${ }^{2}$ Department of Astronomy, Faculty of Mathematics, University of Belgrade, 11000 Belgrade, Serbia
}

Accepted 2016 November 18. Received 2016 November 17; in original form 2016 September 21

\begin{abstract}
The Hoffmeister family is a C-type group located in the central main belt. Dynamically, it is important because of its interaction with the $v_{1 \mathrm{C}}$ nodal secular resonance with Ceres, which significantly increases the dispersion in inclination of family members at a lower semimajor axis. As an effect, the distribution of inclination values of the Hoffmeister family at a semimajor axis lower than its centre is significantly leptokurtic, and this can be used to set constraints on the terminal ejection velocity field of the family at the time it was produced. By performing an analysis of the time behaviour of the kurtosis of the $v_{W}$ component of the ejection velocity field $\left[\gamma_{2}\left(v_{W}\right)\right]$, as obtained from Gauss' equations, for different fictitious Hoffmeister families with different values of the ejection velocity field, we were able to exclude that the Hoffmeister family should be older than 335 Myr. Constraints from the currently observed inclination distribution of the Hoffmeister family suggest that its terminal ejection velocity parameter $V_{\mathrm{EJ}}$ should be lower than $25 \mathrm{~m} \mathrm{~s}^{-1}$. Results of a Yarko-YORP Monte Carlo method to family dating, combined with other constraints from inclinations and $\gamma_{2}\left(v_{W}\right)$, indicate that the Hoffmeister family should be $220_{-40}^{+60}$ Myr old, with an ejection parameter $V_{\mathrm{EJ}}=20 \pm 5 \mathrm{~m} \mathrm{~s}^{-1}$.
\end{abstract}

Key words: celestial mechanics - minor planets, asteroids: general-minor planets, asteroids: individual: Hoffmeister.

\section{INTRODUCTION}

The Hoffmeister family was identified in Milani et al. (2014), and more recently in Nesvorný, Brož \& Carruba (2015) with a Family Identification Number equal to 519. As originally observed by Novaković et al. (2015), this family is characterized by its interaction with the $v_{1 \mathrm{C}}$ nodal secular resonance with Ceres, whose effect is to significantly spread the distribution in inclination of family members for a semimajor axis lower than $\simeq 2.78$ au. Carruba \& Nesvorný (2016) also identified this family as one of the eight groups characterized by having the most leptokurtic distribution of the $v_{W}$ component of the terminal ejection velocity field, which is closely related to the inclination through the third Gauss equation (see for instance, equation 3 in Carruba \& Nesvorný 2016). While most families are formed with an originally leptokurtic distribution of ejection velocities [see Carruba \& Nesvorný (2016) for an explanation of the role that escape velocities from a parent body have in creating originally leptokurtic distributions of terminal ejection velocities], in the absence of dynamical mechanisms able to change the inclination of family members, the distribution of $v_{W}$ tends in time to become more mesokurtic, or Gaussian. This, however, is not the case for the Hoffmeister family, whose interaction with the $v_{1 \mathrm{C}}$ nodal secular resonance significantly shaped its inclination distribution.
The peculiar nature of the Hoffmeister family allows for the use of techniques of family dating not available for other asteroid groups. In particular, the study of the time behaviour of the kurtosis of the $v_{W}$ component of the ejection velocity field $\left[\gamma_{2}\left(v_{W}\right)\right]$, as performed by Carruba (2016) and Carruba et al. (2016) for the Astrid, Gallia, Barcelona and Hansa families, could provide invaluable constraints on the family age and on the $V_{\mathrm{EJ}}$ parameter describing the standard deviation of the initial ejection velocity field, assumed as Gaussian. By observing by what time the current value of $\gamma_{2}\left(v_{W}\right)$ is reached, for fictitious Hoffmeister families with different values of $V_{\mathrm{EJ}}$, and by imposing constraints on the most likely value of this parameter, based on the current inclination distribution of the part of the Hoffmeister family not affected by the $v_{1 \mathrm{C}}$ secular resonance, we can then obtain constraints on the most likely values of both family age and $V_{\mathrm{EJ}}$, not available for regular families.

\section{FAMILY IDENTIFICATION AND DYNAMICAL PROPERTIES}

As a first step in our analysis, we selected the (1726) Hoffmeister family, ${ }^{1}$ as identified in Nesvorný et al. (2015) using the hierarchical clustering method (Bendjoya \& Zappalà 2002) and a cutoff

\footnotetext{
${ }^{1}$ For the sake of brevity, the identification number of families discussed in this paper will only be provided once. After that, families will be referred to only by the parent body name.
} 

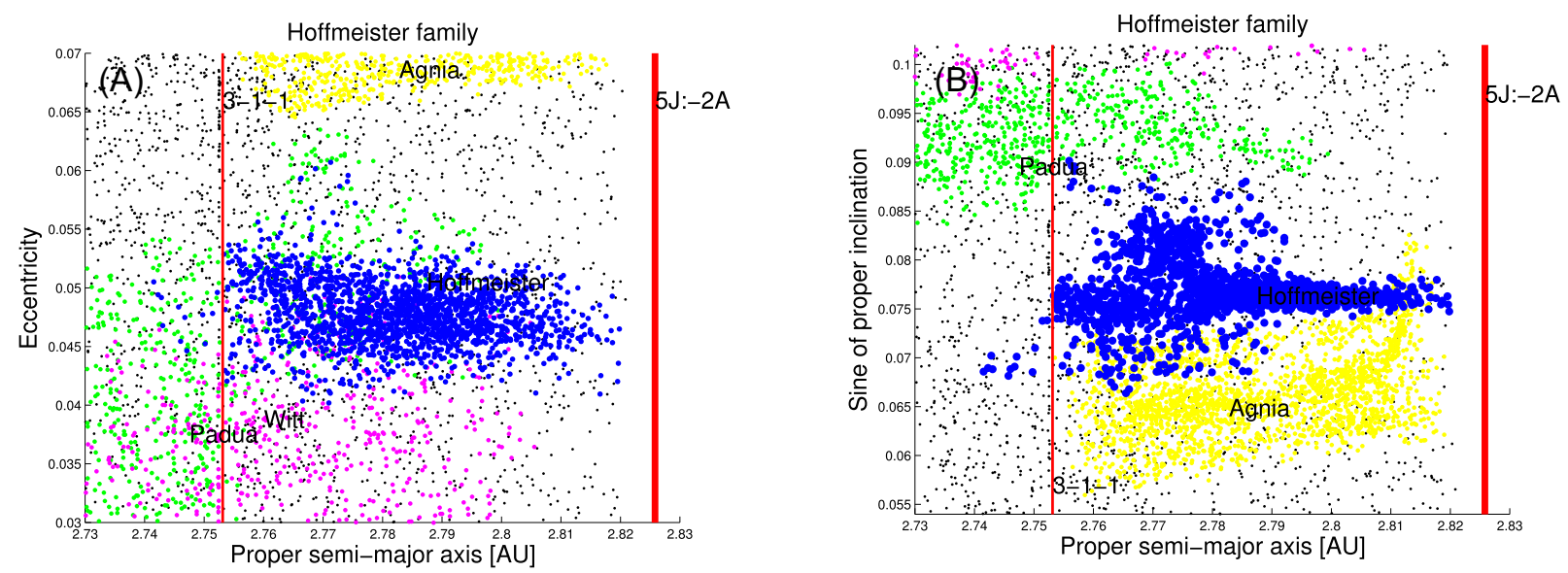

Figure 1. An $(a, e)$ (panel A) and $(a, \sin (i))$ (panel B) projection of asteroids in the local background of the Hoffmeister family. Vertical lines display the location of the main mean-motion resonances in the region. Blue full dots show the orbital location of members of the Hoffmeister family, green dots those of the Padua family, yellow dots those of the Agnia family, and magenta dots are associated with the orbits of members of the Witt family. Black dots show the location of asteroids in the Hoffmeister local background.

of $45 \mathrm{~m} \mathrm{~s}^{-1}$. A total of 1819 members of the Hoffmeister dynamical group were identified in that work. Milani et al. (2014) identified 1905 members of the Hoffmeister family, with an $(a, e)$ and $(a, \sin (i))$ distribution very similar to that observed for the Nesvorný et al. (2015) group. For the sake of consistency, in this work, we will use the data from Nesvorný et al. (2015), but we will discuss any significant difference with results from Milani et al. (2014), when appropriate. Using the criteria defined in Carruba \& Nesvorný (2016), we defined as objects in the local background of the Hoffmeister family those with synthetic proper elements, ${ }^{2}$ whose values of proper $e$ and $\sin (i)$ are in a range from the family barycentre to within four standard deviations of the observed distribution for the Hoffmeister family, namely from 0.0300 to 0.071 in proper $e$ and from 0.054 to 0.102 in $\sin (i)$. The minimum value of proper $a$ was chosen from the minimum value of Hoffmeister members minus $0.02 \mathrm{au}$, the averaged expected orbital mobility caused by close encounters with massive asteroids over 4 Byr (Carruba et al. 2013). The maximum $a$ value was taken at the centre of the $5 \mathrm{~J}$ :-2A mean-motion resonance. Namely, this corresponds to an interval in $a$ from 2.730 to 2.825 au. Overall, we found 4781 asteroids in the local background of the Hoffmeister family so defined, also including known members of other families.

Carruba (2009), in his analysis of the (363) Padua family, found that the main asteroid families in the local background of the Hoffmeister cluster are the (847) Agnia and Padua groups, both characterized by their interaction with the $z_{1}$ secular resonance (Vokrouhlický et al. 2006b; Carruba 2009). This analysis was essentially confirmed by Nesvorný et al. (2015). Apart from the cited Agnia and Padua families, these authors also identified the (2732) Witt family at higher inclinations. Other families in the region, such as the groups of (128) Nemesis, (1668) Hanna and (1222) Tina, do not have family members in the local background of Hoffmeister defined according to our criteria. Concerning the two major other families in the region, Padua and Agnia, despite some difference in terms of family membership between the Nesvorný and Milani groups for the Padua family, which has 1087 and 864 members in these two classifications, respectively; the corresponding

\footnotetext{
${ }^{2}$ We use data from the AstDyS site [http://Hamilton.dm.unipi.it/astdys,
} Knežević and Milani (2003)], accessed on 2016 July 3. distributions in the $(a, e)$ and $(a, \sin (i))$ planes are similar. The only significant difference in the distribution in the $(a, \sin (i))$ plane for the two Padua families is that the Nesvorný group has a small population of objects at $a>2.78$ au not visible in the Milani group. The case of the Agnia family is a bit more problematic. This family has 3033 members in Milani et al. (2014), and only 2125 members in Nesvorný et al. (2015). Most of the difference in family membership is caused by the presence of multiopposition objects in the Milani group, which are not accounted for in the Nesvorný family. Also, the Milani Agnia family extends beyond the 3-1-1 three-body resonance, while the Nesvorný one does not. Quite interestingly, Milani et al. (2014) and Spoto, Milani \& Knežević (2015) identify the (3395) Jitka sub-family inside the Agnia family, which is quite distinguished in terms of physical properties from the rest of the Agnia family, and that will be further discussed in the next section.

These differences in family membership for the Agnia and, in a lesser measure, the Padua groups can a bit affect the determination of the local background but should not change our overall conclusions. Therefore, since there is a good agreement between the two Hoffmeister families, in terms of family membership and other properties, in this work, we will use the families as determined in Nesvorný et al. (2015).

After removing members of the Padua, Agnia and Witt groups, the local background consisted of 1721 objects. Fig. 1 displays a projection as black dots of these asteroids in the $(a, e)$ (panel A) and $(a, \sin (i))$ planes. Members of the Hoffmeister, Padua, Agnia and Witt families are shown as full blue, green, yellow and magenta dots, respectively. As originally observed in Novaković et al. (2015), the left-hand side of the Hoffmeister family is quite more spread in inclination than its right-hand side because of the interaction of this family with the $v_{1 \mathrm{C}}=s-s_{\mathrm{C}}$ linear secular resonance with Ceres. To start assessing the dynamical importance of this resonance, we obtained a dynamical map of synthetic proper elements, with the method discussed in Carruba (2010), for particles subjected under the gravitational influence of all planets, plus Ceres, Pallas and Vesta. ${ }^{3}$ We integrated 3000 particles in a 50 by 60 grid in an

\footnotetext{
${ }^{3}$ Dynamical maps in the $(a, e)$ and $(a, \sin (i))$ planes for the cases without massive asteroids were obtained for the region of the Padua family in Carruba (2009). Interested readers could find more information about these results in that paper.
} 

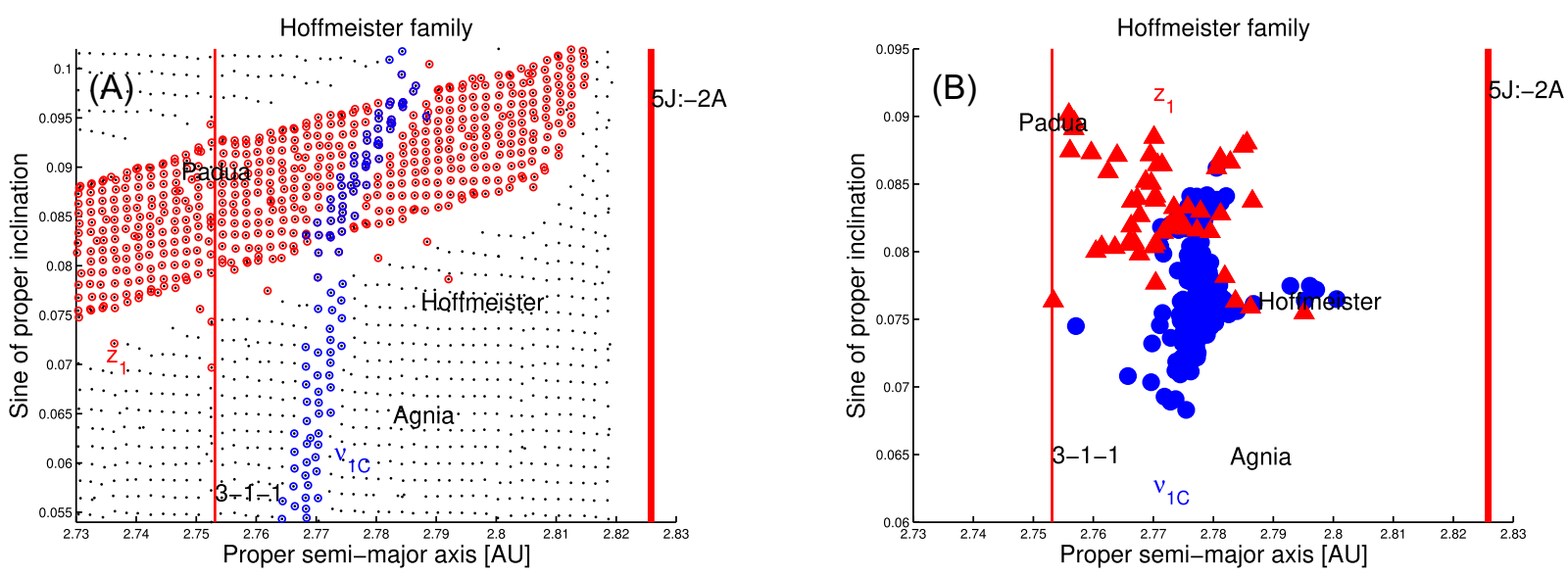

Figure 2. A dynamical map in the proper $(a, \sin (i))$ domain for the orbital region of the Hoffmeister family, considering the effect of Ceres, Vesta and Pallas as massive perturbers (panel A). Black dots identify the values of proper $a$ and $\sin (i)$ for the integrated test particles. Blue and red full dots are associated with likely resonators of the $v_{1 \mathrm{C}}$ and $z_{1}$ secular resonances, respectively. Other symbols are the same as in Fig. 1. Panel B displays an $(a$, sin $(i))$ projection of Hoffmeister members in $v_{1 \mathrm{C}}$ (blue full dots) and $z_{1}$ (red full triangles) librating states, respectively.

osculating initial $(a, \sin (i))$ plane, with a step of 0.02 au in $a$ and 0.1 in $i$. Initial values of $a$ and $i$ were 2.73 au and $0^{\circ}$, respectively. The eccentricity and the other angles of the test particles were those of (1726) Hoffmeister at J2000.

Our results are shown in Fig. 2(A). Values of the proper $(a, \sin (i))$ of each particle are shown as black dots. Blue and red full circles are associated with likely resonators of the $v_{1 \mathrm{C}}$ and $z_{1}$ secular resonances, defined as objects whose $s$ and $g+s$ frequencies are within $\pm 0.2 \operatorname{arcsec} \mathrm{yr}^{-1}$ from the proper node frequency of Ceres for the $v_{1 \mathrm{C}}=s-s_{\mathrm{C}}$ resonance $\left(s_{\mathrm{C}}=-59.17 \operatorname{arcsec} \mathrm{yr}^{-1}\right.$, Knežević and Milani 2003), and from the sum of the proper pericentre and node frequencies of Saturn for the $z_{1}=g+s-g_{6}-s_{6}$ resonance $\left(g_{6}+\right.$ $s_{6}=1.90 \operatorname{arcsec} \mathrm{yr}^{-1}$ Knežević and Milani 2003). As discussed in Carruba (2009), the $z_{1}$ resonance plays a major role in the dynamical evolution of the Padua family, while $v_{1 \mathrm{C}}$ has a pivotal role in the evolution of the Hoffmeister group (Novaković et al. 2015). To check for the effects of these two resonances on the dynamical evolution of the Hoffmeister family, we also integrated 1819 members of the family and checked the time behaviour of the $v_{1 \mathrm{C}}$ and $z_{1}$ resonant arguments over $20 \mathrm{Myr}$. We found 180 and 54 members of the family in librating states of these two resonances, respectively. Their ( $a$, $\sin (i))$ projection is shown in Fig. 2(B). Hoffmeister members are dispersed in inclination after interacting with the $v_{1 \mathrm{C}}$ resonance, as originally observed by Novaković et al. (2015). A significant fraction of Hoffmeister members (about 3 per cent) reached values of inclination large enough to allow for capture into the $z_{1}$ resonance. We expect that some of the past members of the Hoffmeister family could have been drawn to the region of the Padua family and be current interlopers of that group.

\section{PHYSICAL PROPERTIES}

After revising the effect of the local dynamics, we now turn our attention to the taxonomic properties of local objects. Carruba (2009) discuss properties of asteroids in the orbital proximity of the Padua family; interested readers could find more information in that paper. Concerning objects in the background of the Hoffmeister family, as defined in this section, we found 287 objects with photometric data in the Sloan Digital Sky Survey-Moving Object Catalog data, fourth release (SDSS-MOC4; Ivezić et al. 2001), in this region, 71 of which are members of the Hoffmeister dynamical family.
If we consider all available data, regardless of its error, $1515 \mathrm{ob}-$ jects have geometric albedo and absolute magnitude information in the Wide-field Infrared Survey Explorer (WISE) and NEOWISE data bases (Masiero et al. 2012). Fig. 3(A) displays asteroids with their classification obtained with the method of DeMeo \& Carry (2013). Fig. 3(B) displays objects with WISE geometric albedo $p_{V}$ with values compatible with a C-complex taxonomy $\left(p_{V}<0.12\right.$, blue full dots) and with an S-complex taxonomy, $\left(0.12<p_{V}<\right.$ 0.30, red full dots, Masiero et al. (2012). As can be seen from the figure, the Hoffmeister family is compatible with a C-type composition, the Padua family was probably originated from the break-up of an X-type asteroid and most of the members of the Agnia family are S-type, which also confirms the analysis of Nesvorný et al. (2015). As discussed in Spoto et al. (2015), the Jitka sub-family in the Agnia family is characterized by higher albedo values than the rest of the family. While most of Agnia members have $p_{V}=$ $0.15 \pm 0.01$, members of the Jitka sub-family have $p_{V}=0.31$ \pm 0.04 . The Witt family, not visible in the figure, is an S-type group. The mean albedo value for the Hoffmeister, Padua and Agnia families is $(0.05 \pm 0.025),(0.07 \pm 0.03)$ and $(0.15 \pm 0.01)$, respectively.

Concerning halo objects, in the local background of the Hoffmeister family, only the Hoffmeister family itself is associated with a C-type composition. It is reasonable therefore to conclude that the 95 asteroids with an SDSS-MOC4 C-type compatible composition in the region (see Fig. 3) could all be potentially associated with the Hoffmeister family. C-type objects in the region of the Padua family could be potential former members of the Hoffmeister family that reached this region with the mechanism of dynamical diffusion (capture in the $v_{1 \mathrm{C}}$ resonance and then in the $z_{1}$ ) discussed in the previous section. If we consider the albedo data, Fig. 4(A) displays a histogram of the WISE albedo data for the asteroids members of the Hoffmeister (blue line), Padua (green line) and Agnia (red line) families. Vertical red-dashed lines show the $1 \sigma$ range of albedo values for the Hoffmeister family. There is a region of overlapping for asteroids belonging to the Hoffmeister and Padua families (at $1 \sigma$ level the Padua family covers the range of $p_{V}=0.07 \pm 0.03$ ), so that it is not possible to distinguish objects originating from one of these two families based only on albedo (the contribution from the Agnia family in this $1 \sigma$ interval is essentially negligible). If we limit our data to an asteroid in the $1 \sigma p_{V}$ interval $\left(0.025<p_{V}<0.075\right.$, Fig. 4 

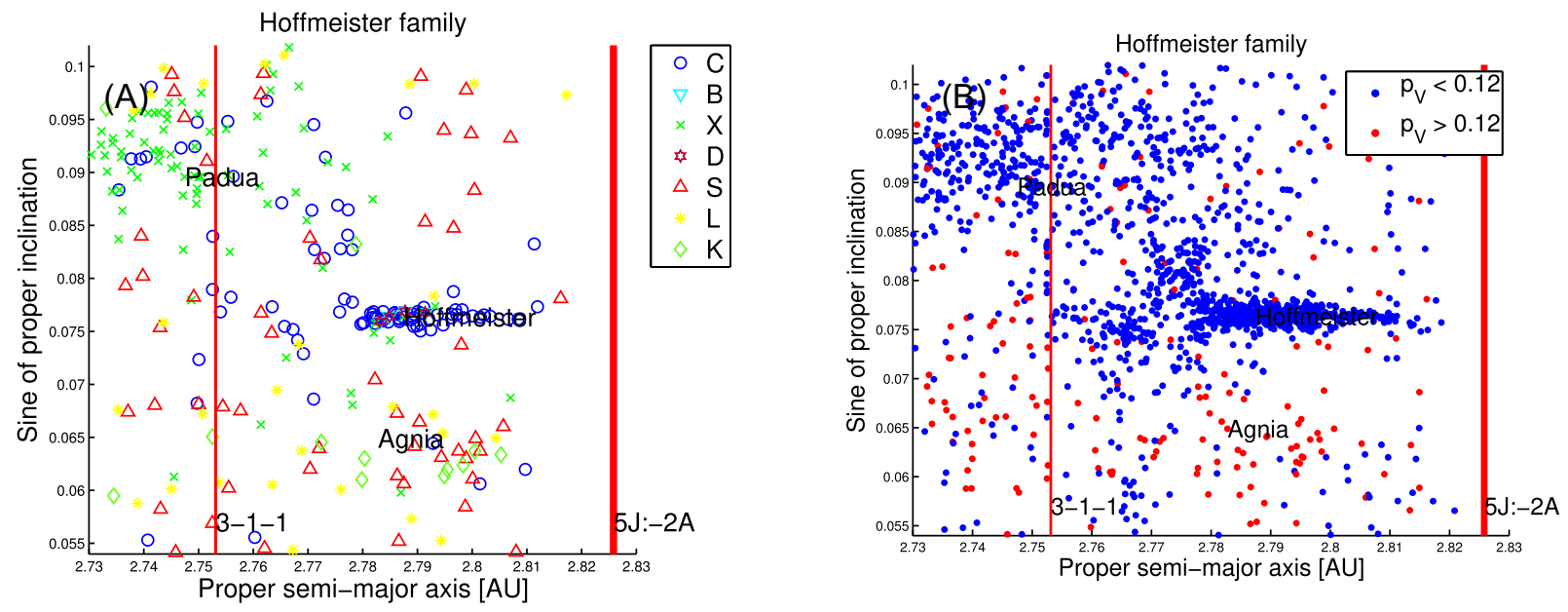

Figure 3. An $(a, \sin (i))$ projection of asteroids with an SDSS-MOC4 taxonomic classification, according to the DeMeo \& Carry (2013) method (panel A). Symbols identify the different asteroids according to the scheme outlined in the figure legend. Panel B displays an $(a$, $\sin (i))$ projection of asteroids with WISE albedo $p_{V}$ lower than 0.12 (full blue dots) and higher than 0.12 (full red dots).
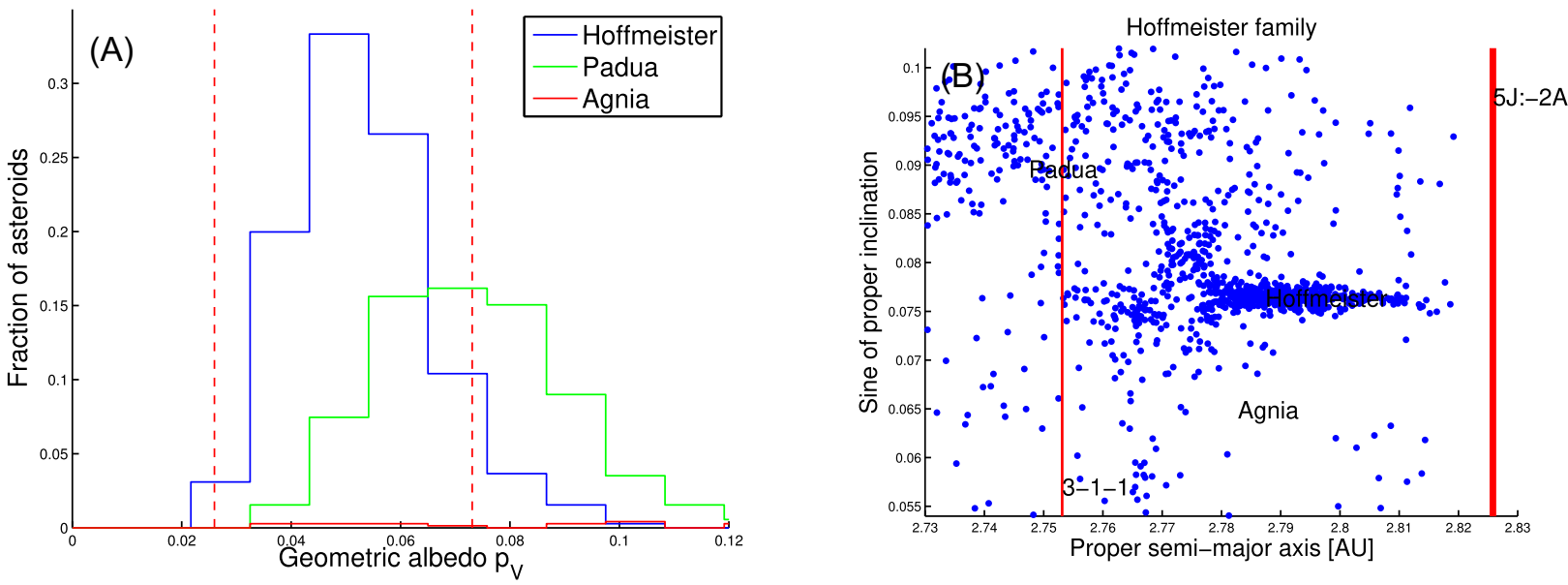

Figure 4. A histogram of the WISE albedo data for the asteroids members of the Hoffmeister (blue line), Padua (green line) and Agnia (red line) families (panel A). Panel B displays an $(a, \sin (i))$ projection of objects with WISE albedo in the $1 \sigma$ interval associated with the Hoffmeister family.

B), then we can safely conclude that objects at an inclination lower than that of the Hoffmeister barycenter should most likely originate from this family. However, no conclusion could be safely reached for objects at higher inclinations.

\section{CONSTRAINTS ON TERMINAL EJECTION VELOCITIES FROM THE CURRENT INCLINATION DISTRIBUTION}

Nesvorný et al. (2015) estimate the age of the Hoffmeister family to be $210 \pm 10 \mathrm{Myr}$, while Spoto et al. (2015), using a V-shape criteria, evaluate the family to be $330 \pm 100 \mathrm{Myr}$ old, and found no asymmetry between the left- and right-hand sides of the family semimajor axis distribution, concerning the estimation of the group age. As discussed in Carruba (2016), Monte Carlo methods (Vokrouhlický et al. 2006a, Vokrouhlický et al. 2006b,c) that simulate the evolution of the family caused by the Yarkovsky and YORP effects, where YORP stands for the Yarkovsky-O'Keefe-Radzievskii-Paddack effect, could also be used to obtain estimates of the age and terminal ejection velocities of the family members (these models will be referred to as 'Yarko-YORP' models hereafter).
However, the age estimates from these methods depend on key parameters describing the strength of the Yarkovsky force, such as the thermal conductivity $K$ and bulk and surface density $\rho_{\text {bulk }}$ and $\rho_{\text {surf }}$, which are in many cases poorly known. Before attempting our own estimate of the family age and terminal ejection velocity field, here we analyse what constraints could be obtained on the possible values of terminal ejection velocities of the original Hoffmeister family from its current inclination distribution.

Assuming, in the first approximation, that the original ejection velocity field of the Hoffmeister family could be approximated as isotropic [see Carruba \& Nesvorný (2016) for a discussion of the caveats on this hypothesis], we can model the distribution of ejection velocities of asteroids with a Gaussian distribution whose standard deviation follows the relationship

$V_{\mathrm{SD}}=V_{\mathrm{EJ}} *(5 \mathrm{~km} / D)$,

where $V_{\mathrm{EJ}}$ is the terminal ejection velocity parameter to be estimated and $D$ is the asteroid diameter. Brož et al. (2013) estimated that the ratio of the radius of the parent body to that of the largest fragment was 0.14 , which yields an estimate of $90.85 \mathrm{~km}$ for the parent body diameter, and an escape velocity of $39.6 \mathrm{~m} \mathrm{~s}^{-1}$, assuming a mean density for the parent body of $1300 \mathrm{~kg} \mathrm{~m}^{-3}$, typical of C-type 

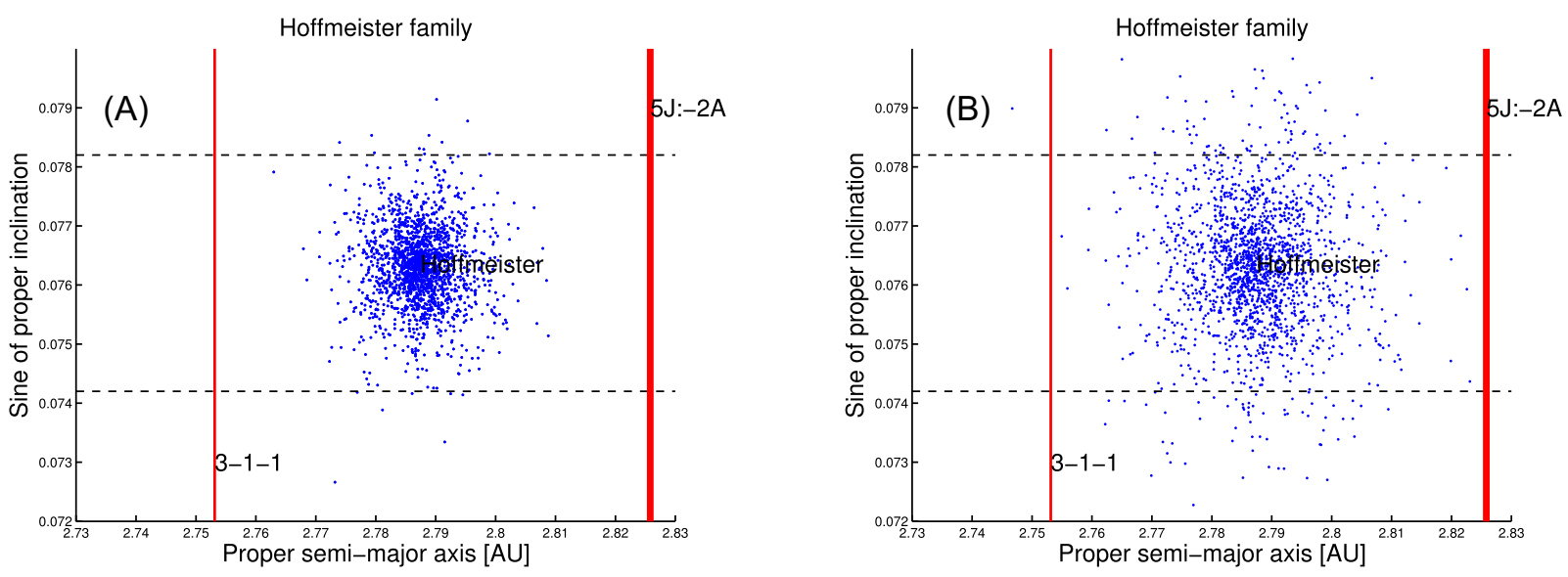

Figure 5. An $\left(a, \sin (i)\right.$ projection of the initial orbital dispersion of a family generated with $V_{\mathrm{EJ}}=15 \mathrm{~m} \mathrm{~s}^{-1}$ (panel A) and $V_{\mathrm{EJ}}=25 \mathrm{~m} \mathrm{~s}{ }^{-1}$ (panel B). The dashed lines show the minimum and maximum values of $\sin (i)$ currently observed for members of the Hoffmeister family with $a>2.795$ au, i.e. those that did not yet interact with the $s-s_{\mathrm{C}}$ secular resonance. The other symbols have the same meaning as in Fig. 1.

asteroids. If we consider only objects with $a>2.795 \mathrm{au}$, so as to eliminate the asteroids that interacted with the $s-s_{\mathrm{C}}$ resonance, then the currently observed minimum and maximum values of $\sin (i)$ of family members are 0.0742 and 0.0782 , respectively. Neglecting possible changes in $\sin (i)$ after the family formation, which is motivated by the fact that the local dynamics do not seem to particularly affect asteroids in this region (see Fig. 2), these values set constraints on the possible terminal ejection velocity parameter $V_{\mathrm{EJ}}$ with which the family was created. We generated synthetic families for values of $V_{\mathrm{EJ}}$ from 5 to $40 \mathrm{~m} \mathrm{~s}^{-1}$. Fig. 5 shows an $(a, \sin (i))$ projection of the initial orbital dispersion of the members of the family generated for $V_{\mathrm{EJ}}=15$ (panel A) and $25 \mathrm{~m} \mathrm{~s}^{-1}$.

For $V_{\mathrm{EJ}}=15 \mathrm{~m} \mathrm{~s}^{-1}$, six particles $(0.3$ per cent of the total) had values of $\sin (i)$ outside the range of values currently observed and $a>2.995 \mathrm{au}$, while for $V_{\mathrm{EJ}}=25 \mathrm{~m} \mathrm{~s}^{-1}$, this number was 48 ( 2.7 per cent of the total). Based on these considerations, it seems unlikely that the ejection velocity parameter $V_{\mathrm{EJ}}$ was larger than $25 \mathrm{~m} \mathrm{~s}^{-1}$, or a larger number of asteroids outside the Hoffmeister family at $a>2.795$ au would be visible today.

\section{EJECTION VELOCITY EVOLUTION}

As previously discussed, the Hoffmeister family is one of the seven families identified in Carruba \& Nesvorný (2016) as being characterized by having a very leptokurtic distribution in the orthogonal component $v_{W}$ of the currently estimated terminal ejection velocities, and, therefore, of the asteroid inclinations. As observed in Section 2, this is caused mainly by the interaction of this family with the $v_{1 \mathrm{C}}$ secular resonance, which strongly affects the distribution in proper inclination of family members at lower values of semi-major axis, and, therefore, their $v_{W}$ values. Recently, Carruba (2016) investigated how the time evolution of $v_{W}$ values could be used to set constraints on the initial values of the $V_{\mathrm{EJ}}$ parameter for the Astrid family. Using the same approach, here we simulated fictitious Hoffmeister families with the currently observed sizefrequency distribution, and values of the parameters affecting the strength of the Yarkovsky force typical of C-type asteroids according to Brož et al. (2013), i.e. bulk and surface density equal to $\rho_{\text {bulk }}=\rho_{\text {surf }}=1300 \mathrm{~kg} \mathrm{~m}^{-3}$, thermal conductivity $K=$ $0.01 \mathrm{~W} \mathrm{~m}^{-1} \mathrm{~K}^{-1}$, thermal capacity equal to $C_{\mathrm{th}}=680 \mathrm{~J} \mathrm{~kg}^{-1} \mathrm{~K}^{-1}$, Bond albedo $A_{\mathrm{Bond}}=0.02$ and infrared emissivity $\epsilon=0.9$. The fictitious families had values of $V_{\mathrm{EJ}}=10$ and $20 \mathrm{~m} \mathrm{~s}^{-1}$, the most likely values of this parameter, according to the analysis of the previous section (values of $V_{\mathrm{EJ}}$ larger than or equal to $25 \mathrm{~m} \mathrm{~s}^{-1}$ were deemed to be incompatible with the current inclination distribution of the Hoffmeister family at large $a$ ). Particles were integrated with SWIFT_RMVSY, the symplectic integrator developed by Brož (1999), which simulates the diurnal and seasonal versions of the Yarkovsky effect, over $400 \mathrm{Myr}$, and the gravitational influence of all major planets plus Ceres. Values of $v_{W}$ were then obtained by inverting the third Gauss equation (Murray \& Dermott 1999):

$\delta i=\frac{\left(1-e^{2}\right)^{1 / 2}}{n a} \frac{\cos (\omega+f)}{1+e \cos (f)} \delta v_{W}$,

where $\delta i=i-i_{\text {ref }}$, with $i_{\text {ref }}$ the inclination of the barycentre of the family, and $f$ and $\omega+f$ assumed equal to $30^{\circ}$ and 50.5 , respectively. Results from Carruba \& Nesvorný (2016) show that the shape of the $v_{W}$ distribution is generally not strongly dependent on the values of $f$ and $\omega+f$, except for values of $\omega+f$ close to $\pm 90^{\circ}$ (which does not seem to be the case for the Hoffmeister family, since these values would have produced a very small inclination distribution).

Our results are displayed in Fig. 6 for members of a fictitious family with $V_{\mathrm{EJ}}=10$ (panel A) and $20 \mathrm{~m} \mathrm{~s}^{-1}$ (panel B). Vertical lines display the maximum range of uncertainty for the family age, according to Spoto et al. (2015), while the horizontal line shows the current value of $\gamma_{2}\left(v_{W}\right)=2.21$ for the Nesvorný et al. (2015) Hoffmeister family [the $\gamma_{2}\left(v_{W}\right)$ for the Milani et al. (2014) family is quite similar and equal to 2.27]. In our computation of $\gamma_{2}\left(v_{W}\right)$, we neglected particles that reached values of $\sin (i)$ beyond $\pm 4 \sigma(\sin (i))$ from the family centre. Individual spikes in the time behaviour of $\gamma_{2}\left(v_{W}\right)$ in Fig. 6 are associated with single particles that temporarily approached such extreme values of inclination. The present value of $\gamma_{2}\left(v_{W}\right)$ is first attained after $320 \mathrm{Myr}$ for the simulation with $V_{\mathrm{EJ}}=10 \mathrm{~m} \mathrm{~s}^{-1}$ and after $260 \mathrm{Myr}$ for the second simulation. It is finally attained at $335 \mathrm{Myr}$ in the first simulation and at $280 \mathrm{Myr}$ in the second, if we do not consider fluctuations associated with isolated spikes. Overall, we have an upper limit for the Hoffmeister age of $335 \mathrm{Myr}$ for $V_{\mathrm{EJ}}=10 \mathrm{~m} \mathrm{~s}^{-1}$ and of $280 \mathrm{Myr}$ for $V_{\mathrm{EJ}}=$ $20 \mathrm{~m} \mathrm{~s}^{-1}$. In the next section, we will try to further refine our family age estimate. 

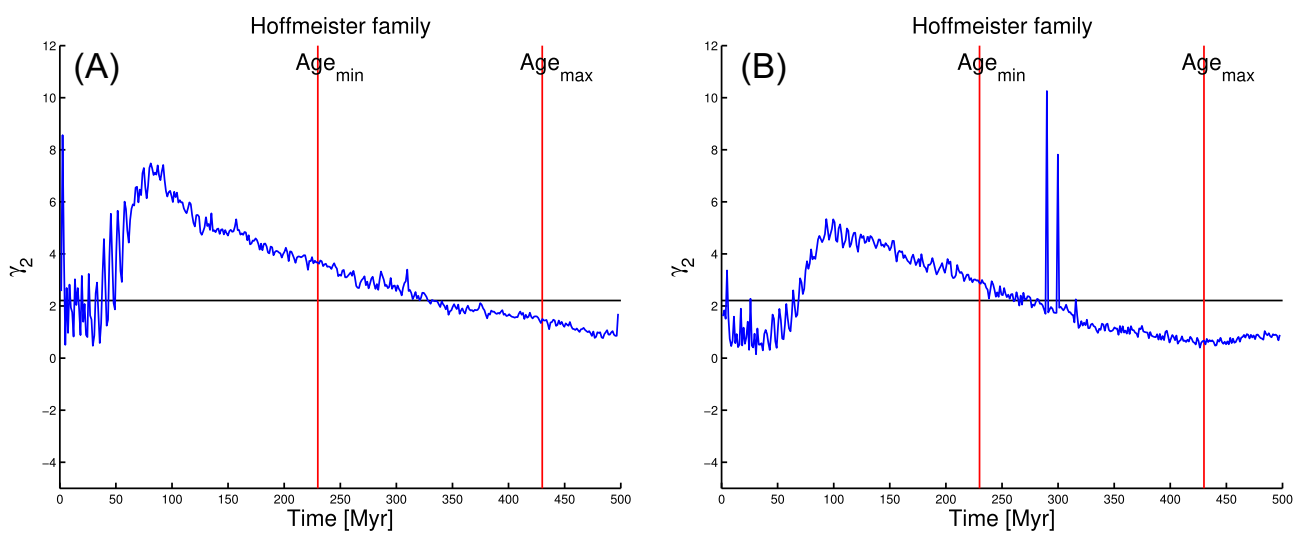

Figure 6. Time evolution of the Kurtosis parameter $\gamma_{2}\left(v_{W}\right)$ for members of a fictitious family with $V_{\mathrm{EJ}}=10$ (panel A) and 20 (panel B) $\mathrm{m} \mathrm{s}^{-1}$. The horizontal line identifies the current value of $\gamma_{2}\left(v_{W}\right)$ for the Hoffmeister family (2.21).
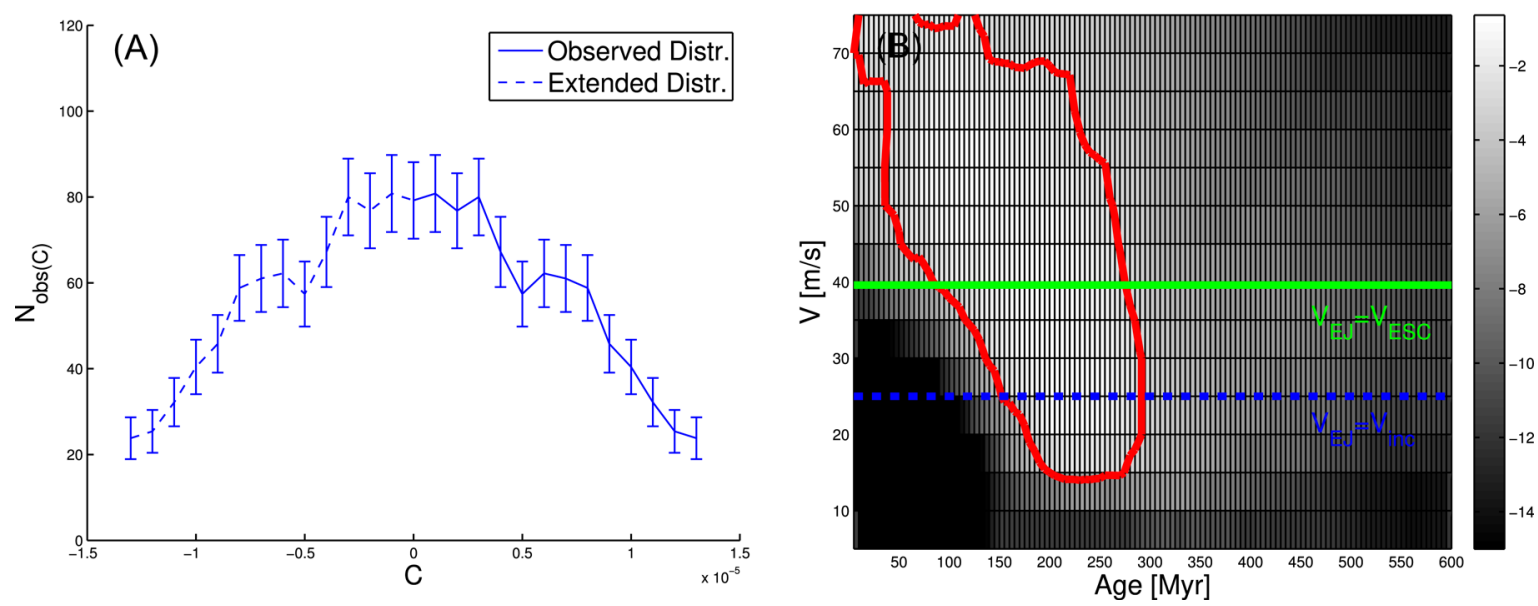

Figure 7. Panel A: histogram of the distribution of $C$ values for the Hoffmeister family (blue line). The dashed blue line displays the negative part of the $C$ distribution. Panel B: target function $\psi_{\Delta C}$ values in the (Age, $V_{\mathrm{EJ}}$ ) plane for a symmetrical bimodal distribution based on the $C$ negative values. The horizontal green line displays the value of the estimated escape velocity from the parent body, while the dashed blue line refers to the $V_{\mathrm{EJ}}=25 \mathrm{~m} \mathrm{~s}^{-1}$ limit obtained from the current inclination distribution in Section 4. The red lines display the contour level of $\psi_{\Delta C}$ associated with a $1 \sigma$ probability that the simulated and real distributions were compatible.

\section{CHRONOLOGY}

Now that the analysis of the current inclination distribution and our $\gamma_{2}$ test provided independent constraints on the values of the $V_{\mathrm{EJ}}$ parameter, we can try to obtain an independent age estimate for this family. We use the approach described in Carruba et al. (2015a), which employs a Monte Carlo method (Vokrouhlický et al. 2006a, Vokrouhlický et al. 2006b,c) to estimate the age and terminal ejection velocities of the family members. More details on the method can be found in Carruba et al. (2015a). Essentially, the semimajor axis distribution of simulated asteroid families is evolved under the influence of the Yarkovsky effect (both diurnal and seasonal versions), the stochastic YORP force and changes in values of the past solar luminosity. Distributions of a $C$-target function are then obtained through the equation

$0.2 H=\log _{10}(\Delta a / C)$,

where $H$ is the asteroid absolute magnitude and $\Delta a=a-a_{\text {centre }}$ is the distance of each asteroid from its family centre, here defined as the family centre of mass. For the Hoffmeister family, this is essentially equal to the semimajor axis of 1726 Hoffmeister itself. We can then compare the simulated $C$-distributions with the observed one by finding the minimum of a $\chi^{2}$-like function:

$\psi_{\Delta C}=\sum_{\Delta C} \frac{\left[N(C)-N_{\mathrm{obs}}(C)\right]^{2}}{N_{\mathrm{obs}}(C)}$,

where $N(C)$ is the number of simulated objects in the $i$ th $C$ interval, and $N_{\mathrm{obs}}(C)$ is the observed number in the same interval. Good values of the $\psi_{\Delta C}$ function are close to the number of the degrees of freedom of the $\chi^{2}$-like variable. This is given by the number of intervals in the $C$ minus the number of parameters estimated from the distribution (in our case, the family age and $V_{\mathrm{EJ}}$ parameter). Using only intervals with more than 10 asteroids, to avoid the problems associated with small divisors in equation (4), we have in our case 14 intervals for $C>0$ [see Fig. 7(A), results from the Milani et al. (2014) Hoffmeister family are compatible to within the errors] and two estimated parameters, and, therefore, 12 degrees of freedom. We use only positive values of $C$ so as to concentrate on the part of the Hoffmeister family at a larger semimajor axis, not affected by the interaction with the $v_{1 \mathrm{C}}$ secular resonance. If we assume that the $\psi_{\Delta C}$ probability distribution follows a law given by an incomplete gamma function of arguments $\psi_{\Delta C}$ and the number of degrees of freedom, the value of $\psi_{\Delta C}$ associated with a $3 \sigma$ 
probability (or 99.7 per cent) of the simulated and real distributions being compatible equals $\psi_{\Delta C}=2.73$ (Press et al. 2001).

Results of our Monte Carlo simulation are shown in Fig. 7(B). If we neglect values of $V_{\mathrm{EJ}}>25 \mathrm{~m} \mathrm{~s}^{-1}$, which are not likely to have occurred based on the current inclination distribution of the part of the Hoffmeister family unaffected by the $v_{1 \mathrm{C}}$ secular resonance, then our best-fitting solution suggests an age of $220_{-40}^{+70} \mathrm{Myr}$ and an ejection parameter of $V_{\mathrm{EJ}}=20 \pm 5 \mathrm{~m} \mathrm{~s}^{-1}$. Combining these results with those of Section 5 (i.e. an age of less than $280 \mathrm{Myr}$ for $V_{\mathrm{EJ}}=$ $20 \mathrm{~m} \mathrm{~s}^{-1}$ ), we can conclude that the most likely age and ejection velocity parameter of the Hoffmeister family are $220_{-40}^{+60} \mathrm{Myr}$ and $V_{\mathrm{EJ}}=20 \pm 5 \mathrm{~m} \mathrm{~s}^{-1}$.

\section{CONCLUSIONS}

Our results could be summarized as follows:

(i) We first identified the Hoffmeister family in the domain of a proper element (Nesvorný et al. 2015), and used the results to define an orbital background region of this group. Other families in the region are the Padua, Agnia and Witt groups. Of the 1819 members of the Hoffmeister dynamical group, 180 asteroids (9.9 per cent of the total) are in librating states of the $v_{1 \mathrm{C}}$ secular resonance, and 54 members (3.0 per cent of the total) are in librating states of the $z_{1}=g-g_{6}+s-s_{6}$. Most of the Hoffmeister $z_{1}$ librators are objects at higher inclinations than those typical for the rest of the family, as also confirmed by our results for dynamical maps in the region (see Fig. 2(A)

(ii) We revised the taxonomic and physical properties of the Hoffmeister family. The Hoffmeister family is compatible with the breakup of a C-type object of low albedo (the mean geometric albedo of the family is 0.05$)$. Objects of low albedo $\left(0.025<p_{V}\right.$ $<0.075)$ at lower inclinations than those of the Hoffmeister family may be former family members. No positive conclusion can be reached for low- $p_{V}$ objects at higher inclinations because of possible contamination from the near X-type Padua family.

(iii) We computed the fraction of particles that would reach regions in sine of inclination incompatible with the current distribution of Hoffmeister members that are not affected by the $v_{1 \mathrm{C}}$ secular resonance, for fictitious families with different values of the $V_{\mathrm{EJ}}$ ejection velocity parameter describing the standard deviation of their ejection velocity field, assumed to be Gaussian. The current inclination distribution of the Hoffmeister family suggests that $V_{\mathrm{EJ}}$ should be less than $25 \mathrm{~m} \mathrm{~s}^{-1}$, or a larger fraction of its members would be currently observed at higher and lower inclinations.

(iv) We studied the dynamical evolution of two fictitious Hoffmeister families under the influence of the Yarkovsky effect for two values of the $V_{\mathrm{EJ}}$ ejection velocity parameter, 10 and $20 \mathrm{~m} \mathrm{~s}^{-1}$. In contrast to the case of the Astrid family, values of thermal conductivity $\left(K=0.01 \mathrm{~W} \mathrm{~m}^{-1} \mathrm{~K}^{-1}\right)$ and mean density $\left(\rho_{\text {bulk }}=\rho_{\text {surface }}=1300 \mathrm{~kg} \mathrm{~m}^{-3}\right)$ appropriate for a C-type class family such as Hoffmeister seem to produce results of $\gamma_{2}\left(v_{W}\right)$ evolution compatible with current estimates of the Hoffmeister family age. Current values of the $\gamma_{2}\left(v_{W}\right)$ parameter for the Hoffmeister family are reached until $335 \mathrm{Myr}$ for $V_{\mathrm{EJ}}=10 \mathrm{~m} \mathrm{~s}^{-1}$ and until $280 \mathrm{Myr}$ for $V_{\mathrm{EJ}}=20 \mathrm{~m} \mathrm{~s}^{-1}$, which sets upper limits on the Hoffmeister family age.

(v) We computed the age of the Hoffmeister family using a Monte Carlo approach for its Yarkovsky and YORP evolution. Our bestfitting solution, also accounting for the results from the $\gamma_{2}\left(v_{W}\right)$ time behaviour analysis, suggests that the Hoffmeister family should be $220_{-40}^{+60}$ Myr old, with an ejection parameter $V_{\mathrm{EJ}}=20 \pm 5 \mathrm{~m} \mathrm{~s}^{-1}$.
Overall, an analysis of the $\gamma_{2}\left(v_{W}\right)$ time behaviour provided invaluable constraints on the age and ejection velocity field of a $v_{W}$ leptokurtic family, such as Hoffmeister, showing once again, in our opinion, the importance that constraints from secular dynamics (in this case, the interaction of the Hoffmeister family with the $v_{1 \mathrm{C}}$ secular resonance) could provide in asteroid dynamics.

\section{ACKNOWLEDGEMENTS}

We are grateful to the reviewer of this paper, Dr Federica Spoto, for comments and suggestions that greatly improved the quality of this work. We would also like to thank the São Paulo State Science Foundation (FAPESP), which supported this work via the grant 16/04476-8, and the Brazilian National Research Council (CNPq, grant 305453/2011-4). BN acknowledges support by the Ministry of Education, Science and Technological Development of the Republic of Serbia, grant 176011. This publication makes use of data products from WISE and NEOWISE, which are a joint project of the University of California, Los Angeles, and the Jet Propulsion Laboratory/California Institute of Technology, funded by the National Aeronautics and Space Administration.

\section{REFERENCES}

Bendjoya P., Zappalà V., 2002, in Bottke W. F., Jr, Cellino A., Paolicchi P., Binzel R. P., eds, Asteroids III. Univ. Arizona Press, Tucson, AZ, p. 613 Brož M., 1999, thesis, Charles University

Brož M., Morbidelli A., Bottke W. F., Rozehnal J., Vokrouhlický D., Nesvorný D., 2013, A\&A, 551, A117

Carruba V., Nesvorný D., Domingos R. C., Aljbaae S., Huaman M., 2016, MNRAS, 463, 705

Carruba V., 2009, MNRAS, 395, 358

Carruba V., 2010, MNRAS, 408, 580

Carruba V., 2016, MNRAS, 461, 1605

Carruba V., Nesvorný D., 2016, MNRAS, 457, 1332

Carruba V., Huaman M., Domingos R. C., Roig F., 2013, A\&A, 550, A85

Carruba V., Nesvorný D., Aljbaae S., Domingos R. C., Huaman M. E., 2015a, MNRAS, 451, 4763

DeMeo F. E., Carry B., 2013, Icarus, 226, 723

Ivezić Ž et al., 2001, AJ, 122, 2749

Knežević Z., Milani A., 2003, A\&A, 403, 1165

Masiero J. R., Mainzer A. K., Grav T., Bauer J. M., Jedicke R., 2012, ApJ, 759, 14

Milani A., Cellino A., Knežević Z., Novaković B., Spoto F., Paolicchi P., 2014, Icarus, 239, 46

Murray C. D., Dermott S. F., 1999, Solar System Dynamics. Cambridge Univ. Press, Cambridge

Nesvorný D., Brož M., Carruba V., 2015, in Michel P., DeMeo F. E., Bottke W., eds, Asteroid IV. Univ. Arizona Press, Tuscan, AZ, p. 297

Novaković B., Maurel C., Tsirvoulis G., Knežević Z., 2015, ApJ, 807, L5

Press V. H., Teukolsky S. A., Vetterlink W. T., Flannery B. P., 2001, Numerical Recipes in Fortran 77. Cambridge Univ. Press, Cambridge

Spoto F., Milani A., Knežević Z., 2015, Icarus, 257, 275

Vokrouhlický D., Brož M., Morbidelli A., Bottke W. F., Nesvorn D., Lazzaro D., Rivkin A. S., 2006a, Icarus, 182, 92

Vokrouhlický D., Brož M., Bottke W. F., Nesvorný D., Morbidelli A., 2006b, Icarus, 182, 118

Vokrouhlický D., Brož M., Bottke W. F., Nesvorný D., Morbidelli A., 2006c, Icarus, 183,349

This paper has been typeset from a $\mathrm{T}_{\mathrm{E}} \mathrm{X} / \mathrm{LT} \mathrm{T} \mathrm{X}$ file prepared by the author. 